\title{
Pratiques contractuelles et individualisation de la rupture de la relation de travail
}

Contractual Practices and the individualized Breach of Employment Relationship

\section{Christian Bessy}

\section{(2) OpenEdition}

\section{Journals}

Édition électronique

URL : http://journals.openedition.org/travailemploi/1932

DOI : 10.4000/travailemploi. 1932

ISSN : 1775-416X

Éditeur

DARES - Ministère du Travail

\section{Édition imprimée}

Date de publication : 15 juin 2008

Pagination : 71-93

ISSN : 0224-4365

\section{Référence électronique}

Christian Bessy, «Pratiques contractuelles et individualisation de la rupture de la relation de travail », Travail et Emploi [En ligne], 114 | avril-juin 2008, mis en ligne le 05 novembre 2010, consulté le 07 avril 2021. URL : http://journals.openedition.org/travailemploi/1932 ; DOI : https://doi.org/10.4000/ travailemploi.1932 


\title{
Pratiques contractuelles et individualisation de la rupture de la relation de travail
}

\author{
Christian Bessy $\left(^{*}\right)$
}

\begin{abstract}
Le recours au licenciement pour motif personnel (LMP), en nette augmentation par rapport au licenciement pour motif économique, est-il uniquement le signe d'une volonté des entreprises de contourner, sans autre détour, les règles trop lourdes qui pèsent en particulier sur le licenciement collectif? N'est-il pas à rattacher plutôt au développement de pratiques contractuelles nouvelles dans les entreprises, visant à individualiser les relations du travail et plus particulièrement la rupture de ces relations? Telle est l'hypothèse de cet article, qui repose sur l'exploitation d'une base de contrats de travail. Il s'interroge principalement sur le régime juridique de la modification du contrat de travail, en pleine évolution particulièrement depuis la loi du 20 décembre 1993, qui a renforcé les garanties individuelles du salarié en cas de modification de son contrat et imposé le recours au licenciement économique, même pour une simple réorganisation du travail, pour le salarié qui ne les acceptait pas. Le recours au LMP s'inscrirait davantage dans une stratégie de contournement de ces garanties, par l'introduction de clauses formalisant l'accord du salarié sur des motifs de rupture et anticipant des litiges éventuels : clauses d'objectifs et de résultats, clauses de variation, clauses de flexibilité fonctionnelle, géographique ou temporelle... Ces clauses préqualifient juridiquement les faits susceptibles d'être reprochés au salarié et préconstituent des motifs de licenciement pour faute, bien que le juge garde en principe le pouvoir d'appréciation de la cause réelle et sérieuse. Elles relèvent des nouvelles politiques de gestion individualisée des RH et de contrôle des performances de l'individu et changeraient graduellement la nature du contrat de travail.
\end{abstract}

En France, le déclin des «licenciements économiques », relativement aux licenciements pour motif individuel (Pignoni, Zouari, 2003), a été souligné par les projets de réforme visant à justifier la réduction de la protection en matière de licenciement pour motif économique. C'est le cas en particulier du rapport CAHUC et KRAMARZ (2005) qui a proposé un contrat unique. Certes, lorsque cette protection est jugée trop contraignante par les employeurs, cela peut induire d'autres modes de rupture ou encore d'autres modes d'engagement dans la relation de travail permettant une plus grande flexibilité, à l'instar du contrat à durée déterminée (CDD). Mais pour expliquer le recours à d'autres modes de rupture du contrat à durée indéterminée (CDI), il semble important de prendre en compte l'ensemble de la transformation des règles juridiques concernant le licenciement. On pourrait par exemple faire référence à l'évolution de l'intervention de l'État (avec un recul relatif des mesures financées par des fonds publics comme les préretraites, la contribution Delalande pour le licenciement économique des salariés âgés...).

(*) IDHE ENS Cachan, CNRS; bessy@idhe.ens-cachan.fr
Cette contribution propose de se focaliser sur les changements jurisprudentiels concernant principalement le régime juridique de la modification du contrat de travail. En effet, notre analyse des pratiques contractuelles (BESSY, 2007), à partir d'une base de contrats de travail, montre que les différentes formes de «contractualisation» du licenciement, repérables dans la rédaction de ces contrats, peuvent être reliées à la politique jurisprudentielle de «restauration du contrat de travail» qui a commencé à se développer à partir de 1987 (LYON-CAEN, 1988), s'est consolidée en 1996 et étendue aux questions disciplinaires en 1998. Nous faisons l'hypothèse que ces nouvelles pratiques contractuelles constituent un facteur explicatif important de recours au licenciement pour motif individuel, en fournissant un appui juridique aux pratiques de gestion individualisée des ressources humaines des entreprises.

Pour étayer cette hypothèse, nous partons du fait que le contrat interindividuel est devenu une source grandissante de droit dans la définition des conditions de travail et que les entreprises mobilisent de plus en plus de ressources pour rédiger les contrats de travail en anticipant les litiges éventuels. Certes, cette tendance à la contractualisation de la relation n'est pas indépendante de la protection en matière 
d'emploi en ce que cette dernière fournit des garanties collectives fortes, en particulier concernant les licenciements pour motif économique. Mais cette contractualisation a aussi sa logique propre articulant une individualisation accrue de la relation de travail, individualisation revendiquée aussi par les salariés, l'accroissement de la recherche de garanties juridiques qui participent à la juridicisation des organisations, au détriment de supports organisationnels reposant sur des garanties plus informelles, et l'intervention croissante des professionnels du droit pour adapter les règles juridiques aux objectifs économiques des entreprises, avocats et juges œuvrant de concert dans la «managérialisation» du droit du travail (EDELMAN, 2003).

Ces différents arguments ont déjà été développés dans notre ouvrage sur La contractualisation de la relation de travail (BESSY, op. cit.), qui fournit une présentation plus étoffée de notre argumentation et des considérations méthodologiques concernant la construction de notre base de contrats. Depuis, des approfondissements supplémentaires ont permis de consolider cette argumentation, en particulier en analysant de façon plus précise le rôle des professionnels du droit (avocats, juristes d'entreprise...) en matière de rédaction des contrats de travail et d'anticipation des litiges. Ce type d'analyse conduit à avoir une conception plus endogène de la règle de droit, comme nous allons le montrer dans le cas du régime juridique de la modification du contrat de travail.

Cette conception endogène de la règle de droit vaut aussi pour analyser de façon plus générale la législation en matière de licenciement. Au lieu de considérer la règle juridique de façon mécaniste, de la réduire à son énoncé, il est important, non seulement, de prendre en compte les interprétations de la jurisprudence, mais aussi, d'étudier comment ces interprétations sont présentées aux gestionnaires des ressources humaines et aux juristes d'entreprise par les avocats et les consultants en organisation. On peut symétriser le raisonnement du côté des avocats qui défendent plutôt les salariés. Mais pour ceux qui défendent les intérêts des employeurs, leur activité de médiation des contraintes juridiques en matière de licenciement contribue à définir les «menaces» que représente le droit, les risques de litige et de perte des procès. Ces professionnels proposent alors des modèles ou des procédures de conformité au droit permettant de réduire ces risques qu'ils ont contribué à construire.

Ce travail de construction de la règle de droit nous éloigne d'une conception du droit considéré comme une contrainte incitative fixée de façon exogène(1). Suivant cette dernière perspective, la

(1) Ce qui est la conception dominante en économie, bien que les travaux les plus en pointe en économie du droit commencent à intégrer le rôle des professionnels du droit et des contraintes de fonctionnement des tribunaux (Deffains, 2008). législation en matière de licenciement, en particulier pour motif économique, est alors présentée comme trop contraignante et elle serait alors contournée comme on cherche à éviter un obstacle naturel qui serait trop coûteux à déplacer. Sans sous-estimer la force incitative de la législation, et donc accepter un minimum de conséquentialisme, il importe de prendre en considération la façon dont est construit son caractère contraignant et les ressources qui sont mobilisées pour organiser son contournement, jusqu'à l'émergence d'une nouvelle norme juridique qui est en train de se dessiner aujourd'hui avec l'accord interprofessionnel du 11 janvier 2008 (DoCKÈs, 2008).

Mais il importe aussi de voir comment cette législation n'est pas simplement contournée mais aussi ajustée aux particularités de différentes configurations productives. En matière de licenciement collectif (au moins dix salariés sur une même période de trente jours), cela renvoie à la codification en la matière opérée par les conventions collectives de branche (BESSY, 1993), par les accords d'entreprises, et, plus récemment, par les accords de méthode (JOBERT, 2008). Suivant cette perspective, la législation ne fournit qu'un cadre juridique général que les partenaires sociaux «travaillent» pour l'adapter à leur contexte productif avec l'aide des professionnels du droit. Plus qu'une contrainte, la règle de droit, ainsi située, apparaît alors aussi comme une ressource qui permet aux acteurs de se coordonner dans la gestion des restructurations (DIDRY, 2000).

Enfin, cette ressource de coordination offerte par un droit des restructurations qui devient de plus en plus procédural, suppose l'existence d'un véritable processus de négociation et donc d'instances représentatives du personnel et des syndicats. En leur absence, les employeurs peuvent effectivement chercher à contourner les contraintes législatives en empruntant d'autres moyens juridiques de rupture des contrats de travail que le licenciement collectif.

En résumé, la législation encadrant les licenciements n'est pas une contrainte dans l'absolu. Contrairement à une approche "mécaniste» de la règle de droit, sa construction et son effectivité vont dépendre de la mobilisation des salariés par l'intermédiaire ou non de leur syndicat, mais aussi du travail des professionnels du droit pour l'adapter à chaque contexte productif. Les asymétries dans les pouvoirs de négociation des parties risquent de neutraliser ces activités de représentation et de médiation, ou de donner lieu à une instrumentalisation du droit au bénéfice de l'employeur qui recherche une certaine sécurité juridique. C'est dans cette perspective que nous allons analyser la «contractualisation» du licenciement.

Dans une première partie, nous présentons l'évolution des règles juridiques concernant le licenciement en lien avec la transformation du régime juri- 
dique de la modification du contrat de travail. Nous examinons la façon dont les acteurs les mobilisent et s'y ajustent dans leurs pratiques contractuelles, en particulier en examinant les effets de certains revirements jurisprudentiels. D'un point de vue empirique, nous mobilisons ici notre base de contrats de travail et nos interviews auprès de professionnels du droit. La seconde partie est consacrée à l'analyse du lien entre ces pratiques contractuelles, les recours à différents motifs de licenciement et l'évolution des litiges du travail, depuis le début des années 1990.

\section{Restauration des mécanismes contractuels et licenciement}

Nous allons revenir dans un premier temps sur l'évolution jurisprudentielle du régime de la modification du contrat de travail, évolution qui nous semble la plus marquante de la transformation de la notion juridique de «contrat de travail». Nous mettons l'accent sur l'utilisation des clauses de flexibilité puis sur tout un ensemble de clauses qui anticipent la rupture de la relation de travail pour faute. L'étude particulière du traitement des clauses d'objectifs par la jurisprudence permet de conforter l'idée d'une contractualisation du licenciement mais aussi d'une évolution de l'objet du contrat du travail qui est de plus en plus associé à des obligations de résultats.

Parallèlement, nous allons examiner ces changements juridiques d'origine jurisprudentielle en lien avec les pratiques contractuelles des entreprises. L'usage de notre base de contrats de travail appelle deux remarques d'ordre méthodologique.

La première porte sur la rédaction du texte des contrats de travail. En effet, au-delà des objectifs poursuivis par les rédacteurs du contrat, en matière de gestion des ressources humaines mais aussi de recherche de garanties juridiques, le degré de formalisme du contrat dépend de la nature de son rédacteur, les avocats étant les plus à même de déployer un certain formalisme en tant que professionnel du contrat, du fait de l'expérience qu'ils ont acquise dans les différents types de transaction encadrés par le droit commun des contrats. Dans notre ouvrage (Bessy, 2007), nous donnons des indications sur les différents rédacteurs des contrats et les ressources qu'ils mobilisent, mais sans avoir eu la possibilité de coder de façon exhaustive le statut du principal rédacteur: responsable des ressources humaines, juristes de l'entreprise, avocat, expert-comptable...

D'après les informations recueillies auprès de responsables de ressources humaines, les avocats sont rarement les rédacteurs à titre principal. Nos interviews auprès d'avocats en droit social montrent que ces derniers jouent un rôle de plus en plus important en la matière, que c'est une activité qui s'est développée à partir des années 1990, donnant lieu à une véritable ingénierie contractuelle en la matière (2). Même si leur intervention directe reste marginale, ils ont néanmoins un rôle prépondérant dans la diffusion de modèles de contrats ou de clauses contractuelles, via les journaux et les revues spécialisés, les séminaires de formation et les clubs de «GRH». Ce rôle d'intermédiation des avocats définissant des modèles de clause contractuelle conformes au droit, est relayé par le travail interne des gestionnaires et juristes d'entreprise visant à concrétiser les «modèles de conformité», à les adapter au contexte organisationnel et aux objectifs d'efficacité définis par les managers. C'est ce qu'un auteur comme L. EDELMAN (2003) analyse comme un processus de «managérialisation» du droit.

L'autre remarque méthodologique concerne notre base de contrats. Elle comprend plus de 400 textes de « contrat de travail» permettant de retracer les pratiques contractuelles avec une certaine représentativité en distinguant trois sous-périodes (1970-1992, 1993-1999, 2000-2004). Nous donnons les caractéristiques de cette base en annexe, et en particulier la façon dont elle a été construite et la justification de la délimitation temporelle retenue. Précisons simplement ici que pour prendre en compte le fait que notre base ne soit pas parfaitement représentative de chaque sous-période, en particulier en matière de catégories hiérarchiques, nous présentons nos résultats, chaque fois que cela a un sens, en distinguant les « cadres» des «non-cadres». Le fait de raisonner uniquement sur la catégorie "cadre» assure en effet une certaine équivalence permettant la comparaison temporelle du fait que ces derniers, au moins sur la période considérée, ont pratiquement toujours signé un contrat de travail écrit.

En résumé, en l'absence de données précises en la matière et du fait de la faiblesse relative de notre échantillon, il s'agit d'un exercice largement exploratoire qui prend appui sur l'hypothèse qu'il existe un lien de causalité, au moins dans certaines configurations, entre l'évolution de la jurisprudence et les pratiques de rédaction des contrats de travail. Nos données contractuelles servent plus à nourrir ce genre d'hypothèse qu'à fournir une base permettant un test statistique de sa justesse.

\section{La transformation du régime juridique de la modification du contrat de travail}

Au tournant des années 1990 (de l'arrêt Raquin de 1987 à l'arrêt Gan-Vie de 1996), l'évolution de la jurisprudence a conduit à réduire le pouvoir de

(2) Une étude en cours sur la réforme de la profession des avocats nous a permis d'approfondir ce point en interrogeant des avocats spécialisés en droit social. 
direction de l'employeur en matière de modification «substantielle» des conditions d'emploi, cette dernière devant recueillir l'accord explicite du salarié. Sous couvert de protéger les salariés contre des modifications de leurs «contrats de travail», la jurisprudence réaffirme la valeur des engagements initiaux et leur force obligatoire. La doctrine sous jacente repose sur l'idée que certaines conditions d'emploi ont une valeur contractuelle et ce même si le salarié peut légitimement croire que cet élément est régi par une source. Cette doctrine, que certains juristes qualifient d'objectivation de la sphère contractuelle (CHRÉTIEN-LESSCHAEVE, 2006), conduit à faire comme si le salaire, le contenu de l'emploi, le lieu et le temps de travail, constituent le socle contractuel minimal, qui ne peut donc être modifié par la suite sans l'assentiment du salarié (WAQUET, 1999). Un corollaire de ce principe jurisprudentiel a été de considérer une parfaite autonomie entre le «statut collectif» et le «contrat de travail». Toute modification du statut collectif sur les éléments essentiels de la relation de travail doit conduire à un avenant individuel au contrat de travail. Dans cette perspective, la négociation de la flexibilité est reportée au niveau collectif, niveau qui permettrait un meilleur équilibrage des intérêts des parties, libre au salarié d'accepter ensuite une modification de ses principales conditions d'emploi.

Nous voudrions montrer dans un premier temps comment ce nouveau régime de la modification du contrat de travail peut être instrumentalisé par les employeurs et plus généralement analyser comment les acteurs peuvent s'ajuster aux règles jurisprudentielles. Dans un second temps, nous présentons nos résultats statistiques susceptibles de corroborer ce genre d'interactions entre droit et économie, entre la mobilisation de règles juridiques et la poursuite d'objectifs de gestion.

\section{Les étapes du processus d'instrumentalisation du droit en matière de modification du contrat de travail}

Pour rendre compte analytiquement de ce processus d'instrumentalisation du droit au bénéfice de l'employeur, on peut distinguer quatre étapes.

\section{La production de standards contractuels par la jurisprudence}

Dans un premier temps, pour donner une ligne directrice aux magistrats dans l'application du nouveau régime de la modification du contrat de travail, la Cour de cassation a été amenée à fixer des standards en matière de contrat de travail et, en premier lieu, en ce qui concerne ce qui est ou n'est pas élément du contrat de travail, ce qui a pour conséquence de limiter le pouvoir d'interprétation des clauses des juges du fond (FAVENNEC-HÉRY, 2004). Ainsi, une règle d'interprétation sur ce qui est un élément du contrat du travail, et donc susceptible de recueillir l'assentiment du salarié, est définie par la Cour et s'impose progressivement aux juridictions inférieures puis à l'ensemble des professionnels du droit: avocats et conseils juridiques.

\section{Les clauses de flexibilité comme ajustement et définition de l'état du droit}

Mais si cette production jurisprudentielle de standards contractuels accroît d'une certaine façon la sécurité juridique en donnant des garanties aux acteurs, elle peut donner prise, dans un second temps, à un usage stratégique du droit par l'introduction dans les contrats écrits de "clauses de flexibilité». Ces clauses correspondent à toutes les mentions du style « sans que cela entraîne une modification substantielle du contrat de travail » accompagnant l'attribution de fonctions ou la définition du lieu de travail ou du temps de travail, ou encore d'un élément de rémunération. Dans cette phase d'ajustement à la nouvelle norme jurisprudentielle, les professionnels $\mathrm{du}$ droit, et en particulier les avocats comme nous l'avons souligné, jouent un rôle essentiel car ils sont les principaux pourvoyeurs de modèles de clauses de flexibilité qui, tout en contournant les contraintes imposées par le principe de l'intangibilité du contrat, respectent néanmoins d'autres principes contractuels, et en l'occurrence ici la possibilité de contractualiser des conditions de travail flexibles. C'est dans ce sens que nous parlons d'instrumentalisation du droit par les employeurs, au moment de la rédaction du contrat de travail, en profitant le plus souvent du faible pouvoir de négociation du salarié et de leur position de pouvoir instituée. Nous voulons aussi signaler par là une asymétrie, au bénéfice de l'employeur, dans la maîtrise de l'interprétation de certaines clauses par les juges (Lascoumes, Serverin, 1995).

\section{La limitation des clauses de flexibilité par la jurisprudence}

Mais cette visée instrumentale, et en particulier l'anticipation d'un licenciement plus aisé dans le cas où le salarié n'accepte pas l'application de la clause de flexibilité, n'a pas échappé au juge. Dans un troisième temps, les litiges générés par l'intégration de clauses de flexibilité dans le contrat de travail vont conduire à un usage plus risqué de ces clauses. Progressivement, la jurisprudence intervient pour limiter l'insertion des «clauses de flexibilité ou de variation» qui permettent à l'employeur de modifier de façon unilatérale certains éléments du contrat de travail (RADÉ, 2001). En particulier, la jurisprudence conditionne l'exécution des clauses de ce genre à l'«intérêt de l'entreprise» ou met en avant la protection des libertés individuelles des salariés en réfé- 
rence à la loi du 30 décembre 1992(3). L'employeur est autorisé à apporter des restrictions aux droits de la personne et aux libertés du salarié que si elles sont justifiées et proportionnées. Le juge cherche alors à trouver un équilibre entre l'«intérêt de l'entreprise» et les contraintes de vie personnelle du salarié.

Par ailleurs, et afin de trouver un compromis entre flexibilité et intangibilité du contrat, la jurisprudence a été conduite à définir des critères beaucoup plus larges en matière de définition des conditions d'emploi et de leur modification (RAY, 2001). Ainsi en matière de définition du contenu de l'emploi, seul un changement de qualification est considéré comme une modification du contrat de travail. En matière de lieu de travail, il doit faire l'objet d'une définition expresse et le critère a été élargi à la définition d'un secteur géographique. Enfin, en ce qui concerne le temps de travail, seule la durée du travail constitue un élément contractuel alors que les horaires de travail et les heures supplémentaires ressortiraient du pouvoir de direction de l'employeur. Leur modification n'exige donc pas l'accord explicite du salarié.

\section{L'extension du nouveau régime aux questions disciplinaires}

Enfin, cette «contractualisation» du pouvoir de l'employeur peut s'observer dans l'évolution de la jurisprudence récente concernant les sanctions disciplinaires conduisant à une modification des conditions de travail. Cette jurisprudence procède à une forme d'unification du régime de la modification du contrat de travail, que cette dernière ait lieu au titre du pouvoir de direction ou du pouvoir disciplinaire de l'employeur. En effet, en application du principe d'intangibilité du contrat, la jurisprudence a conduit à rendre obligatoire l'accord explicite du salarié pour les modifications de son contrat de travail induites par une sanction disciplinaire telle que la rétrogradation ou la mutation (arrêt Hôtel Le Berry du 16 juin 1998). En cas de refus de la sanction disciplinaire, le salarié peut alors être licencié mais garde le bénéfice de ses indemnités. Ce qui constitue une solution plus protectrice du salarié que dans la configuration antérieure, au moins depuis 1996, dans laquelle la jurisprudence considérait que le refus de la sanction par le salarié était constitutif d'une faute grave.

Cette jurisprudence défendue par A. Mazeaud (2003), a été sévèrement critiquée par des auteurs comme C. RadÉ (1999) ou J. Mouly (2002, 2003) au titre qu'elle entretient une confusion des genres entre pouvoir de direction et pouvoir disciplinaire

(3) En suivant en cela J. LE GoFf (2004), on peut voir dans cette loi de 1992, qui a repris l'esprit de la loi d'août 1982 (lois «Auroux») sur les droits individuels fondamentaux (expression des salariés, protection de la vie personnelle et principe de dignité), une source juridique d'individualisation de la relation de travail. de l'employeur. En effet, ce dernier ne peut pas être contractualisé même si la sanction disciplinaire entraîne une modification importante du contrat de travail. Par ailleurs, cette jurisprudence risque de conduire l'employeur à la facilité et à licencier le salarié, alors qu'il aurait peut-être souhaité se contenter d'une sanction d'une moindre envergure. Enfin, elle encouragerait le recours à des clauses de flexibilité à titre disciplinaire (4).

\section{La multiplication des clauses de flexibilité dans les contrats de travail}

Quel est l'apport de notre base de contrats en la matière? Certes, nos données statistiques en évolution sur les clauses de flexibilité ne permettent pas de saisir ce processus d'instrumentalisation de façon précise. Ce que nos données permettent de saisir (tableau 1), c'est avant tout l'augmentation des «clauses de flexibilité» quelle que soit leur source (loi, «statut collectif», ou contrat).

Notons qu'en premier lieu, cette augmentation peut correspondre à un accroissement des contraintes de formalisme imposées par la loi ou par la jurisprudence en renforçant l'obligation d'écriture (FAVENNEC-HÉRY, 2004), y compris pour les clauses ne faisant que reprendre les dispositions de la convention collective (polyvalence, mobilité géographique ou professionnelle (5)...). Ainsi la proportion de clauses de polyvalence pour la catégorie «non-cadres» passe de 7,7\%, durant la période 1970-1992, à $16,1 \%$, au cours de la période 1993-1999, et à 23,9\%, après 2000. Cette augmentation peut être reliée à la codification de la polyvalence dans les grilles de définition des emplois des conventions collectives, en référence à la «logique compétences » (REYNAUD, 2001).

L'augmentation des clauses de flexibilité horaire est à connecter avec la croissance des emplois à temps partiel, emplois pour lesquels, depuis la loi du 20 décembre 1993, il est obligatoire d'introduire dans le contrat de travail une clause qui prévoit les conditions de changement de la répartition de la durée du travail. Si on prend en compte uniquement les contrats à temps complet, les clauses de flexibilité horaire augmentent au cours de la période: de $9,4 \%$ à $12,6 \%$ et $31,2 \%$. De leur côté, les clauses de «forfaits horaires", concernant plus particulièrement la catégorie «cadres», sont en nette augmentation: de $10,7 \%$ à $25,8 \%$ et $27,9 \%$.

(4) Ce qui sera d'ailleurs conforté par une jurisprudence récente (arrêt SA Franfinance du 11 juillet 2001). Sur ce point voir J. Mouly (2002).

(5) D'après les conventions collectives que nous avons examinées, ce type de clause n'est pas codifié en tant que telle, même s'il peut être rappelé que la mobilité constitue un élément important de l'emploi. Ce sont les obligations de l'entreprise accompagnant la mobilité (prise en charge des frais de déménagement pour la mutation géographique, ou des frais de formation pour le changement de fonctions) qui sont codifiées à ce niveau. 
Tableau 1 : Les clauses de flexibilité en évolution (en \%)

\begin{tabular}{|c|c|c|c|c|}
\hline & Ensemble & 1970-1992 & 1993-1999 & 2000-2004 \\
\hline \multicolumn{5}{|l|}{ Flexibilité de l'emploi } \\
\hline Polyvalence (non-cadres) & 17,9 & $7,7 *$ & 16,1 & 23,9 \\
\hline Flexibilité fonctionnelle & 22,8 & 7,1 & 18,2 & 29,8 \\
\hline Flexibilité fonctionnelle (cadres) & 28,0 & 5,9 & 18,6 & 39,8 \\
\hline \multicolumn{5}{|l|}{ Flexibilité géographique } \\
\hline Mobilité géographique & 39,2 & 23,2 & 37,9 & 44,2 \\
\hline Mobilité géographique (cadres) & 54,3 & 17,7 & 48,6 & 65,9 \\
\hline \multicolumn{5}{|l|}{ Flexibilité temporelle } \\
\hline Flexibilité horaire & 34,0 & 12,5 & 32,6 & 40,5 \\
\hline Flexibilité horaire (temps complet) & 22,3 & 9,4 & 12,6 & 31,2 \\
\hline Forfait horaire & 24,8 & 10,7 & 25,8 & 27,9 \\
\hline Référence à des accords d'entreprise dans le contrat & 21,6 & 8,9 & 10,6 & 31,6 \\
\hline
\end{tabular}

* Lecture: parmi les contrats de la période 1970-1992, 7,7\% de ceux concernant les salariés «non-cadres» intègrent une clause de polyvalence. Cette proportion est de $17,9 \%$ pour l'ensemble de la période.

Signalons que ces clauses de flexibilité temporelle ont été le plus souvent négociées par la voie des accords collectifs d'entreprise, en particulier suite aux lois «Aubry» sur la réduction du temps de travail. Notons que ces accords sont en augmentation croissante et plus particulièrement après l'année 2000, 31,6\% des contrats de cette sous-période y faisant référence. L'introduction dans le contrat de travail de ces dispositions issues de la négociation collective ne fait donc que rappeler le contenu de cette négociation sur l'aménagement et la réduction du temps de travail.

Au-delà de ces obligations d'écriture légale ou jurisprudentielle, on ne peut pas faire abstraction des pratiques d'instrumentalisation du droit par l'employeur pour accroître son pouvoir de direction en matière de définition des principales conditions d'emploi. Cela est particulièrement vrai pour les clauses introduisant de la flexibilité fonctionnelle et qui ne sont pas codifiées dans des accords collectifs, comme peuvent l'être des obligations de polyvalence. On peut donner ici l'exemple du contrat d'une «négociatrice» dans une banque. Après avoir rappelé le caractère évolutif du poste la mention poursuit de la manière suivante: «En conséquence, vous pourrez être amenée, en fonction de ces impératifs, à une adaptation corrélative de vos fonctions et vous vous engagez à entreprendre tous efforts en ce sens et, le cas échéant, à accomplir toute formation que nous pourrions être amenés à vous demander.»

Au-delà de l'évolution des fonctions, la clause stipule également une obligation de formation qui pèse sur la salariée.

Pour l'ensemble, ces clauses, visant à accroître la flexibilité fonctionnelle, passent de $7,1 \%$ pour la période 1970-1992 à 18,2\% pour la période 19931999 et $29,8 \%$ après 2000 . Cette augmentation est encore plus significative pour les cadres car la proportion passe de $5,9 \%$ à $18,6 \%$ et $39,8 \%$.

Nos résultats montrent également que les clauses de mobilité géographique augmentent progressivement au cours des trois périodes distinguées: de $23,2 \%$ à $37,9 \%$ et $44,2 \%$ pour 1 'ensemble, et, respectivement, de $17,7 \%$ à $48,6 \%$ et $65,9 \%$ pour les cadres.

Par ailleurs, signalons que les clauses de flexibilité concernant la structure de la rémunération sont peu présentes dans notre échantillon puisque nous avons relevé que 4 cas (renvoyant à la sous-période 1993-1999) dans lesquels le rédacteur du contrat s'est ajusté au standard jurisprudentiel avec une clause du type « vous acceptez le caractère évolutif des critères de détermination de votre rémunération variable et vous ne sauriez dès lors invoquer une modification substantielle de votre contrat de travail...».

\section{La complexité du droit}

On peut donc conclure à un certain ajustement des rédacteurs du contrat par rapport aux standards jurisprudentiels, à une certaine forme d'instrumentalisation du droit au bénéfice de l'employeur, en particulier avec l'introduction de clauses de mobilité géographique et professionnelle qui accroissent son pouvoir de direction. L'usage de ces clauses a été néanmoins limité par la jurisprudence afin de trouver un bon compromis entre intangibilité et flexibilité du contrat. Un juriste comme C. RADÉ (2001) montre que si ce nouveau régime jurisprudentiel de la modification du contrat de travail offre certaines garanties aux salariés, en réduisant le pouvoir de direction de l'employeur, il en souligne le caractère chaotique. Loin d'être définis de façon abstraite, les éléments du contrat de travail sont souvent délimités d'une manière opportuniste, rendant difficile toute 
tentative de systématisation de la jurisprudence et, par suite, imprévisible la solution des litiges.

Une réponse que l'on peut faire à la position de ce juriste est que l'opportunisme du tribunal peut constituer une forme de retour à l'envoyeur, et en l'occurrence ici à l'opportunisme initial de l'employeur. En effet, le juge peut être amené à adopter une règle qui est avantageuse pour la partie la moins bien informée des conséquences de telle ou telle clause intégrée dans le contrat. En rétablissant cette asymétrie d'information (d'interprétation), il contribue à une plus grande complexité du droit, limitant les possibilités d'anticipation des acteurs et donc la recherche d'une certaine sécurité juridique. Mais, ce que nous voulons souligner, c'est que la complexité du nouveau régime de la modification du contrat du travail, dénoncée par le rapport de VIRVILLE (2004), n'est pas seulement liée à une forme de prise de pouvoir par les juges dans la fabrique du droit, elle tient aussi à l'usage stratégique qu'en font les acteurs.

Les avocats eux-mêmes ont bien conscience de cet usage stratégique et certains d'entre eux s'interdisent d'ailleurs de représenter à la fois les employeurs et les salariés. En effet, il est facile de repérer les failles du droit d'un côté et de les exploiter de l'autre côté de la relation de travail, conduisant à un usage de plus en plus stratégique du droit. $\mathrm{Ne}$ pas faire monter systématiquement le contentieux est considéré par certains avocats comme une règle déontologique, mais d'autres, d'après eux, ne s'embarrassent pas de telle considération.

\section{La contractualisation du licenciement}

Nous venons de voir comment les clauses de flexibilité pouvaient s'inscrire dans une forme d'instrumentalisation du droit par l'employeur au bénéfice de son pouvoir de direction et de discipline. Dans certains cas, on peut se demander si leur objectif n'est pas de préconstituer un motif de licenciement, en dehors de toute considération de flexibilité. C'est d'ailleurs ce qui a conduit la Cour de cassation à limiter leur usage.

Au-delà de l'utilisation de clauses de flexibilité, d'autres mentions dans les contrats anticipent la rupture de la relation de travail pour faute. Dans ces cas, il s'agit le plus souvent d'importation dans le texte du contrat de dispositions du règlement intérieur ou d'un code de déontologie. Sans se prononcer sur la légalité d'une telle «contractualisation» du pouvoir disciplinaire, on peut y voir une forme d'instrumentalisation du droit par l'employeur qui cherche à préqualifier juridiquement les faits susceptibles d'être reprochés au salarié. Par ailleurs, l'introduction de clauses considérées ensuite comme illicites par les juges ne leur enlèvent pas tout effet comminatoire à l'égard des salariés (JEAMmaud, 1989).

Certaines évolutions jurisprudentielles récentes vont conforter cette instrumentalisation du droit au bénéfice du pouvoir de licenciement de l'employeur, bien que le juge garde le monopole de l'appréciation d'une cause réelle et sérieuse. Nous allons l'illustrer ici par l'usage des clauses d'objectifs. L'examen du contenu des contrats de travail permet ensuite de mettre en évidence différentes formes de contractualisation du licenciement.

\section{Les clauses d'objectifs à des fins de licenciement}

L'évolution de la jurisprudence en matière de clauses d'objectifs donne une bonne illustration d'une forme de contractualisation du licenciement (voir encadré 1).

Bien que la jurisprudence soit tâtonnante sur cette question, les différentes phases de l'évolution de la jurisprudence et de la transformation concomitante des pratiques contractuelles, au regard au moins des cas de litige qui sont remontés devant les tribunaux, témoignent d'une forme d'instrumentalisation du droit au profit du pouvoir de licenciement de l'employeur. En effet, comme en matière de définition des conditions de travail, les rédacteurs des contrats se sont progressivement ajustés à l'état du droit. Dans un premier temps, la contractualisation des objectifs à atteindre par le salarié est présentée comme une protection du salarié contre le pouvoir de direction de l'employeur. Le licenciement pour non-atteinte des objectifs suppose que les objectifs aient été fixés au préalable par le contrat. Puis, dans un second temps, l'employeur instrumentalise ce type de clause à son profit. Il est précisé en plus dans le contrat que la non-atteinte des objectifs constitue une cause de licenciement, contractualisant ainsi le licenciement.

Prenons l'exemple suivant extrait d'un contrat d'un concepteur de sites Web d'une petite SSII, contrat signé en 1999: "Le salarié s'engage à réaliser un chiffre d'affaires HT minimal mensuel de $70000 \mathrm{~F}$. Cet objectif sera réévalué par accord des parties sous forme d'avenant au présent contrat. L'employeur se réserve le droit de modifier au cours du contrat les objectifs imposés au salarié. La nonréalisation de l'objectif constitue sauf circonstance exceptionnelle un motif légitime de rupture du contrat de travail.»

Cette clause vient s'ajouter à la mention d'une prime mensuelle de $6 \%$ du CA HT mensuel réalisé par le salarié. D'une part, elle illustre le fait qu'il est important de distinguer entre le versement d'une prime liée à la performance individuelle et le fait que le salarié soit astreint à atteindre certains objectifs. D'autre part, elle montre que la non-réalisation 


\section{Encadré 1}

\section{L'évolution de la jurisprudence en matière de clauses d'objectif}

La non-atteinte d'un objectif ne peut être de plein droit une cause de licenciement. Plus généralement, le travail de préqualification des faits ou des comportements défaillants conduisant automatiquement au licenciement, dans le texte du contrat de travail ou de la lettre de licenciement, est difficilement admis par la jurisprudence de la Cour de cassation qui rappelle le pouvoir que le juge tient de l'article L. 122-14-3 du Code du travail d'apprécier l'existence d'une cause réelle et sérieuse.

Néanmoins, un auteur comme P. WAQUET (2001) a mis en évidence une certaine tendance jurisprudentielle qui, depuis 1987, suivant une "politique constante de restauration du contrat du travail ", a obligé l'employeur de recourir au contrat puisqu'elle exigeait que les objectifs soient fixés contractuellement pour que leur non-réalisation justifie un licenciement. La solution peut paraître avantageuse pour le salarié car l'employeur ne peut pas modifier unilatéralement les objectifs prévus par le contrat. Ces clauses étaient encadrées et les juges s'assuraient en particulier d'un véritable consentement au sens que le nouvel embauché a suffisamment d'expérience pour savoir s'il peut atteindre les objectifs.

Par ailleurs, les objectifs ne doivent pas être irréalisables; et cela vaut aussi bien pour une détermination contractuelle que pour une définition unilatérale de l'employeur. Ils doivent tenir compte des compétences des salariés et l'employeur doit fournir les moyens au salarié pour les atteindre. Mais, cette protection du salarié a des revers car le seul fait de ne pas atteindre les objectifs fixés contractuellement risque de se suffire à lui-même, redonnant à l'employeur la souveraineté remise en cause par le contrôle judiciaire.

Un auteur comme S. BRISSY (2004) montre que, prenant acte de cette situation, la Cour de cassation est revenue sur la prévision contractuelle des objectifs. Ainsi, l'arrêt du 14 novembre 2000 déclare que la non-réalisation des objectifs, même fixés par le contrat, ne constitue pas à elle seule une cause réelle et sérieuse de licenciement. Cet excès de contractualisation s'apparentait à une clause résolutoire privant le juge de son pouvoir d'apprécier l'existence d'une cause réelle et sérieuse. Elle était de fait contraire à l'article L. 122-14-3 du Code du travail, ainsi qu'à l'article L. 122-14-7 prohibant la renonciation par le salarié à se prévaloir du droit du licenciement. La Cour de cassation redonne à l'employeur son pouvoir de direction, en particulier en matière de définition des objectifs, mais cherche à le limiter en lui imposant toute une série de contraintes sur le réalisme et la cohérence de son jugement. Ainsi, il doit respecter une certaine cohérence dans l'évaluation des qualités du salarié. Ce dernier ne doit pas être rendu responsable de l'influence des aléas extérieurs sur les résultats de son activité.

Mais, surtout, l'employeur doit montrer que la non-atteinte des objectifs, l'insuffisance de résultats, est due soit à une faute imputable au salarié ou soit à son inaptitude professionnelle (arrêt du 3 avril 2001). Pour Brissy, il s'agit d'une précision très importante car cela est cohérent avec une conception du contrat de travail basé sur un lien de subordination, sur une mise à disposition de la capacité de travail du salarié et le contrôle (par l'employeur) sur son comportement qu'elle implique. En revanche, le licenciement basé uniquement sur l'insuffisance des résultats a pour conséquence d'effacer ce lien de subordination juridique et de faire supporter au salarié les risques de l'activité de l'entreprise.

des objectifs par le salarié peut constituer, pour l'employeur, un motif légitime de rupture du contrat de travail. Mais cette préqualification d'un motif de licenciement par l'employeur va être remise en cause par les tribunaux.

En effet, dans un troisième temps, lors d'un arrêt rendu le 20 novembre 2000, la Cour de cassation intervient pour revenir sur l'excès de contractualisation et redonner à l'employeur son pouvoir de direction, mais, en le limitant. En cas de litige, le juge vérifie le caractère «réaliste» des objectifs qu'il a fixé d'un commun accord ou non avec le salarié. On revient à un jugement au cas par cas, comme pour les litiges autour des clauses de flexibilité, ce qui peut conduire les employeurs à limiter l'usage des clauses d'objectifs en l'absence d'une prévisibilité forte de l'issue des procès.

Un autre enseignement de cette jurisprudence (voir encadré 1) est que la qualification des obligations des salariés opérée par les juges s'approche de plus en plus de l'obligation de résultats, entraînant par là même le travail salarié vers le travail indépendant, par l'intermédiaire de l'objet du contrat qui devient une véritable chose.

Que nous apporte l'analyse des contrats de notre base sur ces pratiques de préqualification du motif de licenciement, et, en particulier, dans le cas des clauses qui fixent d'une manière ou d'une autre des objectifs à atteindre par le salarié?

\section{La préqualification d'un motif de licenciement dans la pratique}

Notre base permet en premier lieu de mettre en évidence toutes les mentions préqualifiant des motifs de licenciement (voir tableau 2). La préqualification d'au moins un motif de licenciement se retrouve dans $23,1 \%$ des contrats de notre base et reste constante au cours de la période d'observation, bien que les contrats après l'année 2000 sont légèrement surreprésentés $(24,5 \%)$ et inversement pour les contrats de la période 1993-1999 (20,8\%). Notons que pour la catégorie «cadre», on assiste plutôt à une diminu- 
Tableau 2 : Évolution des clauses préqualifiant un motif de licenciement et individualisant les performances (en \%)

\begin{tabular}{|c|c|c|c|c|}
\hline & Ensemble & 1970-1992 & 1993-1999 & 2000-2004 \\
\hline \multicolumn{5}{|l|}{$\begin{array}{l}\text { Préqualification d'un motif de licenciement } \\
\text { Au moins une mention }\end{array}$} \\
\hline Ensemble & 23,1 & $23,2 *$ & 20,8 & 24,5 \\
\hline Cadres & 21,7 & 35,3 & 16,2 & 22,8 \\
\hline \multicolumn{5}{|l|}{ Mention systématique } \\
\hline Ensemble & 5,2 & 1,8 & 5,3 & 6,1 \\
\hline Cadres & 2,9 & 0,0 & 4,3 & 2,3 \\
\hline \multicolumn{5}{|l|}{ Clauses d'objectifs } \\
\hline Ensemble & 13,9 & 7,1 & 15,2 & 14,9 \\
\hline - Dont clause avec motif de licenciement & 14,3 & 33,3 & 17,6 & 10,3 \\
\hline Cadres & 24,6 & 17,7 & 17,2 & 31,8 \\
\hline \multicolumn{5}{|l|}{ Rémunération individualisée } \\
\hline Ensemble & 28,4 & 19,6 & 30,3 & 28,8 \\
\hline Cadres & 42,8 & 35,3 & 38,6 & 47,7 \\
\hline
\end{tabular}

tion, ce qui peut témoigner de l'émergence d'autres formes de rupture de la relation de travail. Néanmoins, si on distingue une modalité où ce type de pratique contractuelle est systématique, c'est-à-dire par la mention de plusieurs motifs de licenciement $(5,2 \%$ dans l'ensemble de la période), la proportion de contrats augmente au cours des trois sous-périodes: de $1,8 \%$ à $5,3 \%$ et $6,1 \%$ en fin de période. Mais une telle évolution n'est pas très significative du fait de la faiblesse des proportions enregistrées.

D’une façon générale, la préqualification de motifs de licenciement dans le texte du contrat de travail reste assez stable au cours de l'ensemble de la période. Une telle stabilité reste difficile à interpréter et peut résulter de tendances divergentes. De ce point de vue, rappelons qu'une grande part de la période 1970-1992 est marquée par le régime de l'autorisation administrative de licenciement pour motif économique (à partir de 1975) qui sera supprimée par la loi du 30 décembre 1986. Ce qui peut expliquer que, durant cette période, les employeurs cherchent à préqualifier des motifs personnels de licenciement pour échapper à cette contrainte. Une autre ligne d'argument serait que la préqualification des motifs de licenciement a été limitée par la jurisprudence au cours de la période la plus récente, comme en matière de non-atteinte des objectifs.

Dans notre codage statistique, les « clauses d'objectifs » correspondent à toutes les mentions renvoyant de façon générale à des objectifs à atteindre par le salarié. Nous n'avons pas pu opérer de distinction suivant que la définition de ces objectifs ait été faite d'un commun accord ou imposée par l'employeur.

Ces clauses d'objectifs sont en augmentation puisqu'elles passent de 7\%, durant la période 19701992 , à près de $15 \%$ dans les deux périodes suivantes (tableau 2). Pour les cadres, qui sont la principale catégorie concernée par ce type de clause $(90 \%$ des cas), la proportion de clauses d'objectifs passe de $17 \%$ environ pour les deux premières sous-périodes à $31,8 \%$ après 2000 .

Par ailleurs, parmi les contrats utilisant des clauses d'objectifs seulement $14,3 \%$ d'entre eux stipulent une cause de licenciement en cas de non atteinte des objectifs par le salarié. Notons que cette proportion de 33,3\% avant 1992 (en fait, un contrat sur trois) passe à $17,6 \%$ entre 1993 et 1999 et recule légèrement à $10,3 \%$ après 2000 . Bien qu'il soit difficile de tirer des inférences fortes du fait de la faible occurrence observée, on peut interpréter ce recul en référence au revirement de la jurisprudence, au moins à partir de 2000, limitant l'usage de la nonatteinte des objectifs comme cause automatique de licenciement. Ce qui peut expliquer a posteriori le tassement relatif des pratiques de préqualification de motifs de licenciement.

Notre base de contrats permet également de mettre en évidence des évolutions qui concernent plus généralement la «responsabilisation du salarié», en particulier par la mise en place de dispositifs d'objectivation des performances individuelles qui peuvent toujours ensuite servir à asseoir un motif individuel de licenciement, comme le montre le développement récent des pratiques de ranking (Brissy, 2004). La part des clauses indiquant une rémunération des performances individuelles est en progression au cours de la période d'observation, passant de $19 \%$ en début de période à près de $30 \%$ en fin de période. Cette augmentation est vérifiée si l'on prend uniquement en considération la catégorie «cadres», avec une forte hausse après 2000 (de $35,3 \%$ à $38,6 \%$ et à $47,7 \%$ ).

En conclusion, si nos résultats statistiques restent fragiles, ils mettenten évidence une certaine tendance à la contractualisation du licenciement par la multiplication des clauses facilitant le licenciement du 
salarié à titre individuel. Cette tendance est à relier avec l'individualisation des pratiques de gestion des ressources humaines. Par ailleurs, l'hypothèse d'un certain ajustement des pratiques contractuelles relativement à l'état de la jurisprudence sur le licenciement n'est pas à rejeter. De façon plus générale, on peut y voir une évolution du contrat de travail qui se rapproche d'un contrat d'entreprise en intégrant des obligations de résultats ou des motifs de rupture de contrat en cas de non-respect de certaines clauses (Morin, 1998). Ce qui a pour conséquence de remettre en cause la distinction juridique entre les différents motifs de licenciement.

\section{Le déclin relatif des licenciements pour motif économique}

Nous allons partir des résultats précédents pour revenir sur les facteurs explicatifs du déclin, au cours de la période récente, des licenciements pour motif économique relativement aux licenciements pour motif individuel. Notre hypothèse est que ce déclin relatif peut être expliqué en partie par la contractualisation du licenciement, ce qui conduit, dans un premier temps, à revenir sur le caractère contraignant de la législation sur les licenciements pour motif économique. Dans un second temps, nous faisons des liens avec l'évolution du contentieux en matière de licenciement.

\section{Une législation sur les licenciements économiques trop contraignante?}

Au cours de ces dernières années, de nombreux projets de réforme du droit du travail ont proposé de réduire la protection en matière d'emploi. Ainsi le rapport CAHUC et KRAMARZ (2005) a proposé un contrat unique (un CDI), en réduisant parallèlement cette protection. En effet, cette dernière est jugée trop contraignante par les auteurs qui prennent appui sur l'indicateur de protection de l'emploi calculé par les experts de l'OCDE (licenciement individuel, licenciement collectif, conditions de recours au CDD). Suivant cet indicateur (OCDE, 2004), la France occupe une position moyenne par rapport aux autres pays de l'OCDE mais qui s'est dégradée avec la loi de modernisation sociale du 17 janvier 2002. Cette dernière a accru les contraintes pesant sur l'utilisation des CDD et a modifié les règles du licenciement collectif en renforçant l'intervention de l'administration et les dispositifs de consultation et de protection du personnel, ainsi que les contraintes en matière de reclassement. Malgré la suspension de certains articles par la loi du 3 janvier 2003, le contrôle de la validité du plan de sauvegarde de l'emploi a été maintenu (article L. 321-4-1)(6).

(6) Ce contrôle fait suite à la position de la jurisprudence élaborée en la matière au cours des années 1990 et à la fameuse affaire Michelin.
Mais dans les faits, nous disent les auteurs, les règles très contraignantes en matière de licenciement collectif, et, plus généralement, de licenciement pour motif économique, ne sont pas appliquées. Dans le cas français, la preuve en est faite par la faiblesse du recours au licenciement économique relativement à d'autres pays, ainsi que par l'augmentation de la part des licenciements pour motif individuel dans les motifs de «sorties» (PIGNONI, ZouARI, 2003). Suivant les statistiques de l'ANPE portant sur les motifs de «sorties» des demandeurs d'emploi, on assiste à une augmentation de cette catégorie de licenciement (pour «motif personnel») entre 1993 et 2004, et une hausse très nette à partir de 1998 (de 386055 en 1993 à 391672 en 1998 et à 575433 en 2004), alors que les licenciements pour motifs économiques baissent sur toute la période (de 598362 en 1993 à 178851 en 2004).

Ces résultats statistiques sont alors expliqués par CahUC et Kramarz (2005) par un contournement systématique du droit par les entreprises, en particulier, les licenciements pour motif personnel sont fréquemment des licenciements pour motif économique déguisé. Par ailleurs, ils évoquent le recours de plus en plus privilégié aux accords transactionnels, ce qui permet aux employeurs de contourner leur obligation en matière de reclassement.

Notre objectif n'est pas de discuter leur prémisse de départ, à savoir une législation en matière de licenciement pour motif économique trop contraignante, relativement à d'autres pays européens (7). Encore qu'à ce niveau de généralité statistique, il faudrait avoir une idée de la structure des licenciements suivant les motifs dans les différents pays européens. Par ailleurs, les auteurs en arrivent à oublier le caractère relativement contraignant de cette législation sous le régime de l'autorisation administrative du licenciement pour motif économique jusqu'en 1986. Ce qui à l'époque aurait dû donner lieu à des substitutions massives entre les motifs de licenciement, ce qui n'était pas le cas. En 1985 , le nombre de licenciement pour motif économique était sensiblement équivalent à celui des licenciements pour motif individuel, c'est-à-dire aux environs de 430000 (BESSY, 1993).

\section{Contourner l'accroissement des garanties individuelles protégeant les salariés}

(7) Sur ce point, certains des avocats en droit social interviewés, qui traitent de dossiers de restructuration d'entreprise de taille européenne, convergent vers l'idée que bien que la législation française soit particulièrement protectrice en matière de restructuration, les législations des autres pays sont au moins aussi compliquées tout en étant différentes. 
$\mathrm{Au}$ lieu de mettre l'accent sur les contraintes accrues de la législation française en matière de licenciement collectif pour expliquer le déclin des licenciements économiques, nous faisons porter l'attention plutôt sur l'accroissement des garanties individuelles en cas de modification du contrat de travail (voir encadré 2). En effet, la loi du 20 décembre 1993 va entériner le nouveau régime juridique défini par la jurisprudence et contraindre l'employeur à licencier pour motif économique le salarié qui n'accepte pas une modification de son contrat de travail. Ce qui a pour conséquence de mettre en équivalence une simple réorganisation $\mathrm{du}$ travail avec une restructuration profonde de l'entreprise. L'obligation de respecter les garanties individuelles en matière de licenciement pour motif économique pour une simple réorganisation peut alors avoir un caractère très coûteux (ANTONMATTEI, 2006), ce qui peut expliquer la mise en place de stratégies de contournement de ces garanties.

Comme nous l'avons montré dans la première partie, d'autres évolutions jurisprudentielles ont permis aux employeurs, et les professionnels du droit qui les représentent, de contourner ces garanties individuelles par un licenciement disciplinaire ou par un licenciement qui repose de façon générale sur l'incompétence du salarié (voir encadré 2). Les différentes formes de contractualisation du licenciement que nous avons repérées dans notre base de contrats l'attestent. Prenant conscience de ces stratégies de contournement, la Cour de cassation a d'ailleurs cherché à limiter, au cours de la période la plus récente, l'usage des clauses de flexibilité ou de préqualification des motifs de licenciement.

Ces pratiques contractuelles peuvent donc témoigner d'une législation sur les licenciements pour motif économique jugée sans doute trop contraignante et présentée comme telle par certains professionnels du droit. Mais, surtout, leur examen permet d'entrevoir quelles sont les ressources juridiques mobilisées par les entreprises pour mettre en place les stratégies de contournement les moins risquées. D'une part, les modèles de clauses cherchent à s'ajuster à l'état du droit tout autant qu'elles contribuent à le modifier, par un processus progressif de convergence des représentations des avocats et des juges (EDELMAN, 2003). D'autre part, l'analyse du contexte de ces pratiques de contractualisation du licenciement atteste qu'elles sont d'autant plus courantes que le salarié a peu de pouvoir de négociation sur le marché du travail.

\section{Encadré 2}

\section{Les conséquences en matière de licenciement du nouveau régime juridique de la modification du contrat de travail}

Une des conséquences du nouveau régime juridique de la modification du contrat de travail est que le nonrespect par le salarié d'une clause de flexibilité des conditions de travail peut constituer, sous certaines conditions, un motif de rupture du contrat de travail pour faute grave. En revanche, en l'absence de clauses de flexibilité, l'employeur désireux de modifier le contrat de travail du salarié, pour une raison économique, doit recueillir l'assentiment explicite du salarié. Une procédure spéciale de modification du contrat de travail a d'ailleurs été créée par la loi du 20 décembre 1993 (article L. 321-1-2 du Code du travail) qui entérine d'une certaine façon la jurisprudence Raquin (WAQUET, 1999). D'après cette loi, le silence du salarié vaut acceptation de la proposition écrite faite par l'employeur après expiration d'un délai d'un mois. En cas de refus explicite de ce dernier, l'employeur peut recourir à un licenciement individuel pour motif économique mais en respectant toute la procédure, en particulier en motivant sa décision et donc en justifiant une réorganisation nécessaire à la bonne marche de son entreprise, sinon à sa "compétitivité", ce qui peut être particulièrement coûteux.

Sur cette question, un auteur comme P.-H. Antonmatrel (2006) montre que la mise en équivalence des différentes situations économiques pouvant justifier un licenciement pour motif économique peut être source d'inutiles lourdeurs. En effet, pour l'auteur, il y a une différence économique et sociale de taille entre des suppressions d'emploi liées à des difficultés économiques importantes et une modification du contrat de travail inhérente à une simple réorganisation de l'entreprise.

Cette loi de 1993 accroît donc les garanties individuelles en matière de licenciement pour motif économique, garanties ayant déjà été renforcées à la suite de la loi du 2 août 1989, qui, par ailleurs, avait instauré le droit individuel à la conversion.

Parmi les interventions du législateur qui sont venues limiter la position jurisprudentielle en matière de modification du contrat de travail, et ses conséquences en matière de licenciement, on peut noter les deux lois "Aubry" de 1998 et 2000 portant, d'une part, sur le contrat à temps partiel et, d'autre part, sur les incidences de la réduction de la durée du travail sur le contrat. En particulier, n'est pas soumise à la législation sur les licenciements économiques la rupture du contrat des salariés qui auraient refusé une réduction de leur rémunération inhérente à l'abaissement de la durée du travail dans leur entreprise (article L. 212-3 du Code du travail). Mais à l'exception de la durée du travail, toute modification substantielle du contrat est soumise à cette législation, en cas de désaccord du salarié. De plus depuis l'arrêt Majorette du 3 décembre 1993, lorsque cette modification concerne plus de dix salariés, cela doit entraîner une procédure de licenciement collectif.

Du fait de ces évolutions jurisprudentielles, consolidées le plus souvent par la loi, le licenciement pour motif économique peut s'avérer très coûteux. 
De ce point de vue, le travail économétrique cherchant à expliquer les structures contractuelles les plus typiques (Bessy, SzPIRo, 2008), montre que les contrats de travail qui contiennent le plus de clauses de flexibilité et de préqualification de motifs de licenciement sont ceux pour lesquels le pouvoir de négociation du salarié sur le marché du travail est le plus faible (pouvoir estimer à partir d'un indicateur de tension sur le marché du travail par famille professionnelle). Ces contrats sont également corrélés positivement avec l'absence de représentation syndicale dans l'entreprise. Ce type de résultat conforte l'idée que la décision de licenciement pour motif individuel est préparée bien plus loin en amont, dès la signature du contrat de travail par des salariés qui ont peu de pouvoir de négociation individuellement et collectivement.

Mais, encore plus en rupture avec l'approche de Cahuc et Kramarz, ces pratiques contractuelles, anticipant le licenciement pour motif individuel, s'inscrivent plus largement dans les politiques de gestion individualisée des ressources humaines des entreprises et, dans certaines configurations, de contrôle des performances individuelles, conduisant à changer de façon graduelle la nature du contrat de travail. Dans cette perspective, le recours accru aux accords transactionnels comme mode de rupture du contrat de travail (Melot, 2003), n'est pas seulement un mode de contournement du droit, mais aussi un indice de l'individualisation de la relation de travail.

Si ces pratiques d'individualisation de la relation de travail sont dans certains cas volontairement conçues pour affaiblir les syndicats, ainsi que le rôle de la négociation collective, ces derniers sont aussi condamnés à participer à leur codification, non seulement, pour arriver à frayer des compromis, mais aussi, parce que cela correspond sans doute à une aspiration profonde des salariés d'exercer et de voir préserver leurs libertés individuelles. Comme le montre l'analyse des stratégies des entreprises en matière de restructuration (MELOT 2003; PALPACUER et al. 2005), l'usage d'un dispositif juridique de rupture de la relation de travail dépend du degré de mobilisation collective des salariés, avec ou sans l'entremise des syndicats, mais aussi, de l'anticipation des litiges et des recours des salariés ou non devant les tribunaux. Plus en amont, il dépend également du travail des professionnels du droit (avocats, juristes d'entreprise, syndicalistes) pour l'adapter à chaque contexte productif.

Ainsi, la législation encadrant les licenciements pour motif économique n'est pas une contrainte dans l'absolu. Son effectivité va dépendre de la mobilisation des salariés par l'intermédiaire ou non de leur syndicat, ainsi que de la nature des liens de l'entreprise avec l'administration du travail (BESSY, 1993).

De ce point de vue, Cahuc et Kramarz ont beau jeu de rappeler que de toute façon les instances représentatives du personnel ont peu de pouvoir pour s'opposer en amont à des plans de licenciement (comme par exemple en Allemagne où le plan social est soumis à une obligation de négociation), ce qui est à la source ensuite d'un important contentieux très coûteux. Le problème, à notre avis, est que les syndicats ne sont pas assez présents et que leur possibilité de constitution et d'intervention est minée par de nouveaux modes d'organisation du travail et des entreprises.

\section{Des différents motifs de rupture à l'évitement des tribunaux}

Un autre facteur explicatif est que le recours à un mode de rupture ou à un autre peut dépendre de l'anticipation par l'employeur des litiges auxquels il peut donner lieu et des issues des procès. D'un point de vue statistique, on peut essayer d'appréhender les motifs de litiges portés devant les prud'hommes en les rapportant aux licenciements, avec tous les problèmes de cohérence statistique que cela pose. Au cours de la période 1993-2004, la proportion de recours en cas de licenciement pour motif économique est très faible (de $1 \%$ en 1993 à $2,5 \%$ en 2004) alors que le taux de recours pour les autres motifs (essentiellement «motif personnel») est beaucoup plus élevé avec un pic de $41,0 \%$ en 1998 , puis une baisse régulière jusqu'à 25,8\% en 2004 (Munoz Perez, SERVERIN, 2005). Cette baisse est liée à une hausse très nette des licenciements pour motif individuel alors que le recours devant le tribunal reste constant.

À cet égard, Munoz Perez et Serverin (2005) soulignent qu'en matière de risque judiciaire, les employeurs devraient au contraire éviter les licenciements individuels pour motif personnel et privilégier les motifs économiques. De plus, les résultats statistiques donnés par les auteurs sur les issues des procès montrent que les «demandeurs" (tout motif de demande confondu), en l'occurrence ici les salariés, obtiennent un résultat positif (demandes gagnantes ou présumées gagnantes) dans $64,5 \%$ des procédures lancées devant les prud'hommes pour l'année 2003. Le risque pour l'employeur de perdre un procès est donc élevé. Mais, on peut penser que ce risque est calculé et que l'employeur y trouve d'autres compensations (8).

Par ailleurs, on peut chercher à expliquer la baisse du taux de recours devant les prud'hommes suite à un licenciement pour motif individuel, à partir de 1998.

(8) Des auteurs comme Galdon-SAnChEZ, GüEll (2004) ont cherché à modéliser l'arbitrage entre deux motifs de licenciement suivant les coûts anticipés de rupture. Ils montrent qu'il peut être optimal pour une entreprise de déclarer un licenciement disciplinaire (à la place d'un licenciement pour motif économique) même si elle a toutes les chances de perdre le procès. Tout dépend de l'écart entre les indemnités de licenciement économique et les dommages et intérêts à verser si l'entreprise perd son procès pour licenciement (disciplinaire) injustifié. 
En premier lieu, signalons que cette baisse intervient pendant une conjoncture économique favorable jusqu'en 2001 et défavorable après. Après 2001, on peut évoquer l'argument basé sur le fait que les salariés anticipent des décisions qui leur seraient défavorables (MARISNESCU, 2003) (9).

Mais au moins deux autres facteurs explicatifs sont à prendre à compte. D'une part, les employeurs, via un effet d'apprentissage, ont été amenés à mieux ajuster leurs pratiques contractuelles aux règles retenues par les juges, ce qui a permis de réduire le nombre de litiges. Quand ces règles leur sont favorables, comme nous l'avons montré dans la partie précédente à propos de la «contractualisation» du pouvoir disciplinaire, cela peut conduire les salariés, par le biais de leurs avocats, à anticiper des décisions des juges qui ne sont pas à leur avantage.

D'autre part, il se peut que le recours accru aux accords transactionnels ait permis de réduire le contentieux en matière de licenciement pour motif individuel, en particulier à partir de 1996, année qui correspond à l'émergence d'une nouvelle jurisprudence qui conditionne la validité d'une transaction à une décision préalable de licenciement. Comme le montrent les travaux de Mецот (2003), l'augmentation des accords transactionnels a pour conséquence d'augmenter le nombre de décisions de licenciement, au moins devant les administrations compétentes, et de baisser le recours judiciaire; ce qui peut constituer un facteur important de la baisse du taux de recours aux prud'hommes, et pas seulement pour les cadres. En effet, l'auteur montre que la transaction peut être utilisée en série pour les salariés non-cadres dans le cadre d'une gestion systématique des flux de départs, précédant ou non l'annonce d'un «plan social». Le recours à la transaction vise bien plus à garantir la sécurité juridique des ruptures de contrats réalisées en série, qui déguisent un motif économique sous un motif personnel, qu'à régler un litige particulier.

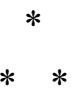

On peut conclure sur l'idée que l'instrumentalisation du droit par les employeurs, orientée vers l'individualisation de la rupture de relation de travail, leur permet, non seulement, de contourner les garanties individuelles de protection des salariés en matière

(9) L'auteur montre que le recours aux prud'hommes serait pro-cyclique au cours des années 1980 et 1990, c'est-à-dire que le taux de recours serait corrélé positivement avec le taux de croissance du PIB réel. L'idée est que les salariés licenciés ont d'autant plus recours aux prud'hommes que la conjoncture économique est bonne et qu'ils anticipent donc des décisions, dans cette configuration, qui leur sont favorables. Inversement, les prud'hommes réduiraient leur taux d'acceptation et les montants de dommage attribués en temps de crise. Nous discutons cette analyse dans Bessy (2007). de licenciement pour motif économique, mais aussi de leur assurer une certaine sécurité juridique. Cette dernière peut être très calculée, l'entreprise espérant néanmoins un gain même si la rupture est source de litige et si l'issue du procès lui est défavorable. Elle peut aussi recourir à la transaction si le salarié a un certain pouvoir de négociation. Mais en l'absence de ce pouvoir de négociation, et plus en amont dans la signature du contrat, les salariés risquent de se faire imposer des clauses qui faciliteront leur licenciement pour motif personnel.

Malgré la fragilité des différentes sources statistiques que nous avons mobilisées, ces pratiques contractuelles peuvent constituer un facteur explicatif du déclin des licenciements pour motif économique relativement aux licenciements pour motif individuel, à partir du début des années 1990. Ce type d'explication a besoin d'être confirmé par une extension de notre base de données. Mais l'intérêt de ce type d'exercice exploratoire est qu'il poursuit une explication sensiblement différente de celles qui mettent uniquement l'accent sur le contournement du droit du licenciement pour motif économique par les entreprises (CAhUC, Kramarz, 2005). Certes, ce droit, jugé trop contraignant, peut expliquer les pratiques d'individualisation de la rupture du contrat de travail. Mais ces pratiques ont des ressorts plus puissants, à la fois juridique, économique et social. Elles dépendent en particulier des pouvoirs de négociation des acteurs économiques, y compris ceux de l'État, et de leur usage du droit. Et c'est le second intérêt de notre analyse que de chercher à mettre en évidence une plus grande juridicisation des rapports de travail qui prend appui sur le «renouveau contractuel» et qui peut donner prise à un usage stratégique du droit, autre qu'un simple contournement.

Enfin, le troisième intérêt de notre analyse est de mettre en évidence une tendance longue témoignant de l'évolution progressive de la catégorie juridique $\mathrm{du}$ «contrat de travail», de l'importance accrue de la figure du contrat, mais aussi, de l'objet du contrat qui est de plus en plus associé à des obligations de résultats. Sur ce point l'accord interprofessionnel du 11 janvier 2008 légalisant d'une certaine façon la rupture du contrat de travail par consentement mutuel (en permettant au salarié de bénéficier des indemnités de licenciement et de chômage) ne constitue que la fin logique d'une lente évolution conduisant à individualiser la rupture de la relation de travail. L'accroissement de la durée de la période d'essai, proposé par l'accord, est aussi une évolution que nous avons constatée, à partir de notre base de contrats, pour toutes les catégories socioprofessionnelles. Elle accroît l'incitation à la performance individuelle, tout comme pourrait le faire le CDD à objet déterminé sur une période de trois ans, réservé aux ingénieurs et aux cadres. D'une façon générale, la limitation de la durée des contrats constitue l'aboutissement de l'objectivation des obligations salariales de résultats. 


\section{Annexe: Aspects méthodologiques et statistiques}

Notre base de données est constituée de 403 textes de «contrats de travail» provenant de plus de 300 entreprises appartenant à différents secteurs d'activité. Ces contrats ont été collectés auprès de différents interlocuteurs: salariés $(31,8 \%)$, DRH $(28,3 \%)$, avocats $(20,1 \%)$, inspecteurs du travail $(11,7 \%)$ et syndicats $(8,2 \%)$.

Le raisonnement en évolution pose immanquablement des problèmes de représentativité de notre échantillon par rapport à la "population-mère» qui comprend l'ensemble des contrats signés entre 1970 et 2004. L'évolution de la catégorie juridique «contrat de travail», ainsi que ce qui peut faire office de preuve de l'engagement contractuel, rend problématique la définition d'une populationmère sur l'ensemble de la période. C'est pour cela que nous avons distingué différentes sous-périodes. Chronologiquement, nous avons retenu en premier lieu l'année 1993 comme une année de référence pour découper notre base. Cela correspond à l'année de mise en application de la directive européenne de 1991 relative à «l'obligation de l'employeur d'informer le travailleur des conditions applicables au contrat ou à la relation de travail» (10). Rappelons que jusqu'à cette date il n'y avait pas d'obligation d'écriture des CDI. On peut penser également que la transformation du régime jurisprudentiel de la modification du contrat du travail est bien stabilisée à partir de cette date, bien qu'il faudra attendre l'arrêt Gan-Vie de 1996. Signalons également la loi de 1990 sur la requalification des CDD en CDI, obligeant à l'écriture des premiers.

L'autre année de référence que nous avons choisie est l'année 2000 qui correspond à la seconde loi «Aubry» sur le passage aux 35 heures. En effet, comme le montrent nos différentes observations (en particulier les interviews auprès de DRH), la négociation collective autour de la réduction du temps de travail a non seulement débouché sur des avenants au contrat de travail, mais aussi, ces avenants ont conduit à la rédaction accrue des contrats de travail, en particulier en introduisant des clauses types suivant le régime horaire choisi par le salarié.

(10) Les mentions obligatoires sont: l'identité des parties, le lieu de travail, la qualification (titre, grade, qualité et catégorie) de l'emploi et sa description sommaire, la date de début de contrat, la durée prévisible du travail s'il s'agit d'un contrat temporaire. Par ailleurs, l'information peut résulter d'une référence aux dispositions législatives, réglementaires, statutaires ou aux conventions collectives applicables, pour: la durée du congé payé, le délai de préavis de fin de contrat, les différents éléments de salaire et la périodicité du versement de la rémunération, la durée du travail, journalière ou hebdomadaire. Si une référence est faite à une convention collective, ou son équivalent, le document écrit remis au travailleur doit lui permettre d'identifier cette convention collective.
Nous avons ainsi distingué trois sous-périodes: l'une allant de 1970 à 1992, qui comprend 13,9\% des contrats, l'autre allant de 1993 à 1999 (32,7\%) et la dernière allant de 2000 à 2004 et regroupant 53,4\% des contrats de notre base. Nous allons donner la composition de notre base de contrats en termes de $\mathrm{CDI} / \mathrm{CDD}$, temps complet/partiel, catégories hiérarchiques et conventions collectives ou secteur d'activité d'appartenance de l'entreprise, car ce sont les caractéristiques les plus déterminantes du contenu des contrats de travail (tableau 3).

C'est parce que les différents types de contrat obéissent à des contraintes de formes différentes, mais aussi correspondent à des choix de gestion de la main-d'œuvre, que nous avons cherché à être «représentatif» en la matière sur l'ensemble de notre échantillon et pour chaque sous-période. Ainsi 17\% des contrats de notre base sont des CDD et $23 \%$ des contrats à temps partiel, ce qui est proche de la moyenne nationale au cours des dernières années pour les premiers ( $11 \%$ en 2000 , enquête «Emploi» de l'INSEE) et pour les seconds (part qui serait de l'ordre de $17 \%$ ). On peut néanmoins noter que les CDD sont nettement surreprésentés pour la période 1970-1992 (17\%), ainsi les que contrats à temps partiel pour la période 1993-1999 (34\%).

Pour les catégories hiérarchiques et les «secteurs d'activité», nous n'avons pas cherché à être «représentatif» dans la constitution de notre base. Notons que la part de contrats signés par des "cadres», au sens des conventions collectives de branche, est nettement surreprésentée (44\%). N'ayant pas de chiffre sur la population mère en ce qui concerne ce mode de classement, on peut rapporter ce chiffre à la part de la catégorie «cadres et professions intellectuelles supérieures» dans la population active totale (non compris les agriculteurs et les artisans), qui est de l'ordre de $15 \%$ en 1999 (dernier recensement de la population). Signalons que cette sur représentation encore plus forte pour la période 1993-1999 (53 \%). Dans notre analyse en évolution, nous avons en partie résolu ce problème en présentant des résultats qui prennent appui uniquement sur cette catégorie.

Enfin, nous avons construit une variable permettant de caractériser la convention collective ou le «statut», dans lequel s'inscrit le contrat de travail. Dans les cas où l'effectif est trop faible nous avons fait des regroupements. En tout, vingt modalités ont été retenues. On peut noter une forte surreprésentation des contrats faisant référence à la convention collective des «bureaux d'études et de conseil» $(12,4 \%)$, en particulier pour la période 1992-1993 $(15,9 \%)$. Par ailleurs, la convention collective de l'Union des industries métallurgiques de la région parisienne est fortement surreprésentée pour la période 1970-1992 (19,6\%). Excepté ces deux conventions collectives, il n'y a pas de variations inconsidérées pour les autres. 
Tableau 3 : Caractéristiques de notre base de contrats de travail (en \%)

\begin{tabular}{|c|c|c|c|c|}
\hline Caractéristiques des emplois & Ensemble & 1970-1992 & 1993-1999 & 2000-2004 \\
\hline \multicolumn{5}{|l|}{ Nature juridique des contrats } \\
\hline CDI & 82,6 & 82,1 & 89,4 & 78,6 \\
\hline \multirow[t]{2}{*}{ CDD } & 17,4 & 17,9 & 10,6 & 21,4 \\
\hline & 100,0 & 100,0 & 100,0 & 100,0 \\
\hline Temps partiel & 23,0 & 5,4 & 34,0 & 20,9 \\
\hline \multicolumn{5}{|l|}{ Catégories hiérarchiques } \\
\hline Ouvriers & 8,2 & 16,1 & 5,3 & 7,9 \\
\hline ETAM & 48,4 & 53,6 & 41,7 & 51,2 \\
\hline \multirow[t]{2}{*}{ Cadres } & 43,4 & 30,3 & 53,0 & 40,9 \\
\hline & 100,0 & 100,0 & 100,0 & 100,0 \\
\hline \multicolumn{5}{|l|}{ Convention collective ou secteur } \\
\hline Statut (anciennes entreprises publiques) & 2,9 & 8,9 & 1,9 & 2,3 \\
\hline Statut professionnel & 2,9 & 3,6 & 3,8 & 2,3 \\
\hline Agro-alimentaire & 3,7 & 3,6 & 3,0 & 4,2 \\
\hline CC UIMM région parisienne & 5,2 & 19,6 & 3,0 & 2,8 \\
\hline $\mathrm{CC}$ ingénieurs et cadres de la métallurgie & 5,7 & 7,1 & 9,8 & 2,8 \\
\hline Autres industries & 7,2 & 8,9 & 6,1 & 7,4 \\
\hline CC commerce denrée alimentaire & 5,4 & 3,6 & 6,0 & 5,6 \\
\hline Autres commerces & 7,7 & 1,8 & 8,3 & 8,8 \\
\hline CC hôtel-café-restaurant & 3,9 & 0,0 & 5,3 & 4,2 \\
\hline Autres activités de restauration & 2,7 & 1,8 & 3,8 & 2,3 \\
\hline $\mathrm{CC}$ transports routiers & 7,0 & 5,4 & 4,6 & 8,8 \\
\hline $\mathrm{CC}$ transports aériens (au sol) & 2,5 & 8,9 & 1,5 & 1,4 \\
\hline $\mathrm{CC}$ banques & 4,7 & 5,4 & 5,3 & 4,2 \\
\hline $\mathrm{CC}$ assurances & 2,7 & 0,0 & 1,5 & 4,2 \\
\hline Autres activités financières & 2,2 & 1,8 & 3,0 & 1,9 \\
\hline $\mathrm{CC}$ bureau d'études et de conseil & 12,4 & 3,6 & 15,9 & 12,6 \\
\hline Autres services aux entreprises & 5,7 & 7,2 & 4,5 & 6,0 \\
\hline Éducation, santé et action sociale & 7,2 & 3,6 & 8,3 & 7,4 \\
\hline Activités récréatives et culturelles & 4,2 & 1,8 & 2,3 & 6,0 \\
\hline \multirow[t]{2}{*}{ Autres services } & 3,7 & 3,6 & 2,3 & 4,6 \\
\hline & 100,0 & 100,0 & 100,0 & 100,0 \\
\hline Total & 100 & 13,9 & 32,7 & 53,4 \\
\hline
\end{tabular}




\section{Bibliographie}

Bessy C. (1993), Les licenciements économiques: entre la loi et le marché, Paris, CNRS éditions.

Bessy C. (2007), La contractualisation de la relation de travail, collection «Droit et Société», Paris, Éditions LGDJ.

Bessy C., SzPIro D. (2008), “The Selection of Provisions in a Labour Contract: an Empirical Investigation of the French Case", document de travail de 1'IDHE, $n^{\circ}$ 01/08, janvier.

BRISSY S. (2004), L'obligation de résultat dans le contrat de travail, thèse pour le doctorat en droit de l'université de Lille II, décembre.

Cahuc P., Kramarz F. (2005), De la précarité à la mobilité : vers une Sécurité sociale professionnelle, rapport au ministre de l'Économie et ministre de l'Emploi, collection «Rapports officiels», Paris, La Documentation française.

Chrétien-Lesschaeve A.-S. (2006), Interprétation et contrat, étude en droit du travail, thèse pour le doctorat en droit de l'université de Nanterre.

DefFains B. (2008), «Introduction à l'analyse économique des systèmes juridiques», Revue économique, volume $58, \mathrm{n}^{\circ} 6$, pp. 1149-1162.

de Virville M. (2004), Pour un code du travail plus efficace, rapport au ministère des Affaires sociales, du Travail et de la Solidarité, collection «Rapports officiels », Paris, La Documentation française.

DiDRY C. (2000) «La règle de droit comme équipement pour le travail juridique: le cas du licenciement collectif pour motif économique», in Kirat T., Serverin E., (éds), Le droit dans l'action économique, Paris, CNRS Éditions, pp. 133-158.

Dockès E. (2008), «Un accord donnant, donnant, donnant, donnant...», Droit Social, no 3, mars, pp. 280287 ,

Edelman L. (2003) "Law at Work: An Institutional Approach to Civil Rights", WP of Center for Advanced Studies in the Behavioral Sciences, Stanford, California.

FAVENNEC-HÉRY F. (2004), «Le contrôle judiciaire du contrat de travail», in La négociation du contrat de travail, Teyssier B. (éd.), Paris, Dalloz.

Jeammaud A. (1989), «Les polyvalences du contrat de travail », in Les transformations du droit du travail, études offertes à Gérard Lyon-Caen, Paris, Dalloz, pp. 299-316.

Le Goff J. (2004), Du silence à la parole, une histoire du droit du travail, Presses universitaires de Rennes.

Galdon-Sanchez J.E., GüEll M. (2004), "Lets Go to Court! Firing Costs and Dismissal Conflicts", Industrial Relation Section, Princeton University, № 444.

Jobert A. (2008), «La négociation collective des restructurations en France: de la consultation des représentants du personnel à la négociation des accords de méthode», Management International, volume 13, nº 2 .

LAsCoumes P., SERVERIN E. (1995), «Le droit comme activité sociale: pour une approche wébérienne des activités juridiques», in Lascoumes P. (éd.), Actualité de Max Weber pour la sociologie du droit, Paris, LGDJ, pp 155177.

LYON-CAEN A. (1988), «Actualité du contrat de travail, bref propos», Droit Social, no 7-8, juillet-août, pp. 540-543.

MARISNESCU I. (2003), L'application du droit du travail est-elle biaisée par les conditions économiques? Working Paper EHESS, LES, CEP, 2003, janvier.

Mazeaud A. (2003), «Contractuel, mais disciplinaire», Droit Social, no 2, février, pp. 164-165.

Melot R. (2003), Entre contrat et procès: enquête sur les transactions entre employeur et salarié, thèse pour le doctorat en sociologie, ENS-Cachan.

Morin M.-L. (1998), «Louage d'ouvrage et contrat d'entreprise», in Le travail en perspectives, Supiot A. (éd.), Paris, LGDJ, pp 101-124.

Mouly J. (2002), «Une remise en cause indirecte de la jurisprudence Hôtel Le Berry: l'utilisation des clauses de mobilité à titre disciplinaire», Droit Social, n ${ }^{0} 11$, novembre, pp. 955-958.

Mouly J. (2003), «Disciplinaire, donc non contractuel», Droit Social, no 4, avril, pp. 395-397.

Munoz Perez B., Serverin E. (2005), Le droit du travail en perspective contentieuse, 1993-2004, rapport pour le ministère de la Justice, DACS, novembre.

OCDE (2004), OECD Employment Outlook, OECD, Paris.

Palpacuer F., Seignour A., Vercher C. (2005), Le licenciement pour motif personnel: un dispositif juridique au carrefour des mutations contemporaines de relation d'emploi, rapport pour la Dares, octobre.

Pignoni M.-T., Zouary P. (2003), «Les nouveaux usages du licenciement pour motif personnel ». Premières Informations et Premières Synthèses, Dares, $n^{0} 28.2$, juillet.

RADÉ C. (2001), «La figure du contrat dans le rapport de travail», Droit Social, no 9/10, pp. 802-812.

RAY J.-E. (2001), «Le noyau dur du contrat de travail», Revue de gestion des ressources humaines, pp. 91-96.

Reynaud J.-D. (2001), «Le management par les compétences, un essai d'analyse», Sociologie du travail, 43, 1, pp. 7-31.

Waquet P. (1999), «Le renouveau du contrat de travail», études et doctrine, Revue de jurisprudence sociale, $\mathrm{n}^{\circ} 5$, pp 383-394.

Waquet P. (2001), «Les objectifs», Droit Social, n 2 , février, pp. 120-125. 